\title{
UM ESTUDO DE CASO DA UTILIZAÇÃO DO MÉTODO MASP PARA MELHORIA DA PRODUTIVIDADE EM UMA LINHA DE PRODUÇÃO DE ALIMENTOS NO AGRESTE PERNAMBUCANO.
}

\author{
Ana Camille da Costa Lins (UFPE) lins.camille@gmail.com \\ Marcos Antonio Sáles Guimarães (UFPE) marcos_asg2_@outlook.com \\ Marcele Elisa Fontana (UFPE) marcele.elisa@ufpe.br \\ José Leão e Silva (UFPE) zeleao@gmail.com
}

\section{Resumo}

Devido à intensa disputa mercadológica nos dias atuais, é de vital importância que uma empresa, que pretende ocupar uma posição de liderança no mercado, tenha uma oferta de produtos ou serviços de qualidade. Porém, para que seja ofertado um serviço com o mínimo de perdas e/ou custos possível, é necessário que as empresas estejam atuando de forma competitiva no mercado, dessa forma a empresa fideliza seus clientes na medida em que gera um padrão de excelência para os seus serviços e processos. Este trabalho apresenta a utilização do método de análise e solução de problemas (MASP) para identificar soluções ao problema de paradas não programadas na produção e consequentes reprocessos de biscoitos em uma empresa localizada no agreste de Pernambuco. Para tal fim, foi utilizado uma gama de ferramentas quantitativas e qualitativas onde o referido problema analisado e solucionado teve uma redução significativa de 53\% nas paradas não programadas referentes a produção de biscoitos.

Palavras-Chaves: MASP; Ferramentas da qualidade; Melhoria.

\section{Introdução}

Em decorrência do aumento populacional, a demanda de bens e serviços tem crescido exponencialmente. Desta forma, mesmo com a crise política e econômica que o Brasil vem passando, o setor de alimentos continua em grande crescimento, a fim de atender a demanda e as necessidades básicas de seus clientes (MARA, MARQUES e ALVES, 2018). Com isso, de acordo com a Associação Brasileira de Indústrias Alimentícias (ABIA), no ano de 2016, o setor industrial alimentício contribuiu em 614,3 bilhões no PIB nacional, representando cerca de 10,1\% (ABIA, 2017).

Contudo, nos últimos anos, os consumidores estão experimentando mudanças imponentes no setor de alimentos em função de vários fatores, entre eles, a globalização, agilidade de informação, hábitos e costumes diferentes (LIMA, DO 
NASCIMENTO e FARIAS, 2016). Ao se deparar com uma nova realidade no mercado, a retenção de custos e maior confiabilidade em um processo tornam-se fundamental e básico para a sua permanência no mercado (MARINO, 2006). Em decorrência disso, as empresas estão em busca de métodos mais sofisticados para a produção e comercialização de seus produtos (DA SILVA e AMARAL, 2004).

A empresa estudada está localizada no agreste de Pernambuco, produzindo café e biscoitos, salgadinhos, massas, wafer e misturas para bolos. Esta empresa vem enfrentando problemas relacionados ao retrabalho da matéria prima decorrentes de alto volume de paradas de linha (não programadas), incluindo ainda, perdas pelo elevado tempo das paradas, bem como, a retomada da produção até que os parâmetros da linha estejam dentro de seus padrões de produção.

Por se tratar de um parâmetro importante para a produção, as paradas de linha não programadas desempenham um impacto significativo na produção: quanto menos paradas, mais rentável será para a empresa, visto que a disponibilidade das máquinas em operação pontua um maior volume de produtividade para a empresa.

Este trabalho objetivou aplicar o Método de Análise e Solução de Problemas (MASP) para identificar soluções ao problema de paradas não programadas na produção e consequentes reprocessos de biscoitos da empresa estudada.

O presente trabalho apresenta características relevantes para a empresa, uma vez que poderá contribuir para a geração de resultados positivos ao processo eliminando as perdas provenientes por erros de processo, permitindo ganhos de produtividade e redução de custos com a possível melhoria dos indicadores, com a oportunidade de alcançar resultados ainda melhores com a continuidade da aplicação da metodologia.

Justifica-se, portanto, que o tema da pesquisa desenvolvida neste trabalho seja pela aplicabilidade de técnicas para melhoria de processo da linha de produção estudada trazendo melhorias para o desenvolvimento e aprimoramento de processos industriais visando melhores resultados em seus indicadores de desempenhos operacionais.

Além desta introdução, este trabalho foi organizado da seguinte maneira: a secção 2 traz alguns conceitos relevantes para contextualizar a pesquisa; na secção 3 a metodologia usada foi descrita; a secção 4 apresenta o estudo de caso; na secção 5 os resultados e sua análise foram relatados; e, por fim, as conclusões foram feitas na secção 6. 


\section{Referencial teórico}

A gestão da qualidade na indústria de alimentos recebeu atenção significativa nos últimos anos, especialmente nos mercados emergentes globais (CHEN, ZHANG e DELAURENTIS, 2014).

Neste sentido, as ferramentas da qualidade são fundamentais no desempenho da qualidade estratégica das empresas, visando extinguir e coibir o surgimento de problemas através de dispositivos, procedimentos gráficos, analíticos, esquemas de funcionamento, formulações gráficas, mecanismos de operações entre outros. Assim possuindo o propósito de apoiar o caminho para se resolver o problema (DE AZEVEDO, COSTA e SILVA, 2018).

Algumas ferramentas básicas da qualidade podem ser destacadas, tais como: Brainstorming; Folha de verificação; Gráfico de Pareto; Diagrama de causa e efeito; Gráfico de controle, Fluxograma e a Análise dos 5 por quês (CARPINETTI, 2012; MACHADO, 2012; SLACK, CHAMBERS e JOHNSTON, 2015; VIEIRA, 2014). Além destes, existem métodos que auxiliam na sistematização do problema, contribuindo no alcance das soluções, tais como o ciclo PDCA e o MASP.

\subsection{Método PDCA}

O ciclo PDCA é um método gerencial para a promoção da melhoria contínua e reflete, em suas quatro fases, a base da filosofia do melhoramento contínuo. Praticando-as de forma cíclica e ininterrupta, acaba-se por promover a melhora contínua e sistemática na organização, consolidando a padronização das práticas (MARSHALL JR., ROCHA e MOTA, 2012).

Este ciclo pode ser enxergado como uma poderosa ferramenta para os programas e processos da qualidade. É ordenado, e tem uma característica muito importante: a flexibilidade a ajustes. Em outras palavras, é uma ferramenta gerencial de tomada de decisões para garantir o alcance das metas necessárias à sobrevivência de uma organização (CAMARGO, 2011).

O método PDCA esta dividido em quatro partes (Serviço Brasileiro de Apoio às Micro e Pequenas Empresas - SEBRAE, [2020]): 
- Plan (Planejar): Definir as metas, as oportunidades de melhorar, as falhas e como resolvê-las;

- Do (Desenvolver): É a execução das tarefas de acordo com o que for estipulado no plano, inclui também a coleta de dados para o controle do processo.

- Check (Verificar): É a fase de monitoramento, medição e avaliação. Deve-se verificar se a meta estabelecida foi alcançada e se as falhas foram sanadas, assim reavaliando também o planejamento.

- Act (Agir): Fase em que se apontam soluções para os problemas encontrados, sendo o ciclo reiniciado tomando-se como base o que foi feito.

\subsection{MASP}

De acordo com Pires (2014), o método de análise e solução de problemas (MASP) possibilita aprimorar competências, habilidades técnicas em prol do aprendizado a fim de solucionar os problemas das empresas.

Campos (2004) relatou que a implementação do MASP consiste em 08 etapas:

1) Identificação do problema: Nesta etapa será definido o problema que será estudado e apresentado, junto com as justificativas que levaram a escolha. Após a escolha, o problema é apresentado e são oferecidas as informações conhecidas a respeito do problema. Nesta etapa é apresentado o período que o problema se refere, as possíveis perdas e ganhos gerados com sua existência e os responsáveis pelos estudos.

2) Observação do problema: Nesta etapa o objetivo é descobrir características a respeito do problema a traves da coleta de dados. Nesta fase não há limite de tempo, pois quanto maior o tempo gasto para observar o problema, menor será o tempo dedicado a resolvê-lo. A análise deve ocorrer no local onde o problema foi identificado para que não aja uma observação distorcida.

3) Analise: Neste processo, são encontradas as causas reais do problema, para facilitar esse processo é utilizado a ferramenta dos 5 porquês, onde são lançadas as causas, permitindo um detalhamento dos possíveis motivos de cada causa apresentada e assim encontrar a causa raiz.

4) Plano de ação: Nesta fase é elaborado o plano que englobe as ações propostas para neutralizar as causas fundamentais do problema, para isto, monta-se uma 
tabela com as seguintes colunas (sugestão): ações propostas; ações sobre causa e efeito; existência de efeito colateral; prazo de implantação e custo de implantação. Para bloquear as causas prováveis, é utilizada a técnica dos 5 porquês, é preciso também estabelecer metas a serem atingidas

5) Execução: Nesta etapa, são divulgados os resultados do MASP e os treinamentos necessários para as pessoas responsáveis possam lidar com o problema.

6) Verificação: Nesta etapa, os resultados e os custos inicias são comparados com os obtidos após a implementação da contra medidas propostas. Então é analisado se ouve algum ganho após a utilização do MASP. Se os efeitos indesejáveis persistirem, significa que a solução foi falha, tão um novo MASP deve ser feito após a implementação das contra medidas (sugere-se um período que não seja inferior a dois meses).

7) Padronização: As instruções utilizadas no processo de desenvolvimento do MASP devem ser testadas e analisadas antes de serem mapeadas, é vital que após o mapeamento dos processos, antigos vícios não tornem a aparecer, incorporando padrões de trabalho que qualquer trabalhador possa realizar a tarefa. Os novos procedimentos devem ser divulgados a todos os envolvidos no processo, expondo razões, motivos e benefícios das mudanças.

8) Conclusão: Relacione se o problema foi resolvido. Os resultados acima da expectativa também devem ser apresentados, pois indicadores de eficiência do estudo podem ser utilizados para possíveis correções dos erros remanescentes, que, devem ser corrigidos para atingir o $100 \%$ de eficiência da meta proposta inicialmente.

Nas etapas do método MASP são utilizadas as ferramentas da qualidade para que possa resolver o problema de forma eficaz.

\section{Metodologia}

A pesquisa caracteriza-se como um estudo de caso em uma pesquisa exploratória, de caráter empírico para explorar uma situação real a partir de evidências dos dados atuais de produção de biscoitos. É um estudo de campo, que será utilizada metodologia de solução de problemas para aplicação prática, apoiada na fundamentação teórica. 
Para o desenvolvimento da pesquisa foi utilizado uma abordagem combinada, ou seja, qualitativa e quantitativa. Qualitativa, pois os dados obtidos são descritivos e sua análise é de forma intuitiva, fornecendo informações sobre as características investigadas. Quantitativa, pois são mensuradas as variáveis do estudo sendo elaborados gráficos e tabelas com os dados obtidos, além de análises e conclusões das informações produzidas.

O Método de Análise e Solução de Problemas (MASP) foi escolhido para atuar nas causas geradoras dos problemas da linha de produção. Assim, o procedimento metodológico consistiu na implementação do MASP. Além disso, é importante mencionar a relação do ciclo PDCA com o MASP, uma vez que a cada ciclo PDCA pode ser visualizado como uma utilização completa da ferramenta MASP. Campos (2004) apresentou um modelo (Figura 2) que relaciona as etapas do ciclo PDCA com as fases do método MASP.

Figura 2 - Procedimento metodológico

\begin{tabular}{|c|c|c|c|}
\hline PDCA & Fluxograma & Fase & Objetivo \\
\hline \multirow{4}{*}{$\mathrm{P}$} & & Identificcação do problema & $\begin{array}{c}\text { Definir claramente o problema e } \\
\text { a necessidade de melhoria }\end{array}$ \\
\hline & & Observação & $\begin{array}{c}\text { Investigar as caracteristicas } \\
\text { especificas do problema }\end{array}$ \\
\hline & & Análise & $\begin{array}{c}\text { Descobrir as causas } \\
\text { fundamentais do problema }\end{array}$ \\
\hline & & Plano de ação & $\begin{array}{c}\text { Conceber um plano para } \\
\text { bloquear as cauas fundamnetais }\end{array}$ \\
\hline $\mathrm{D}$ & & Ação & bloquar as causas fundamentais \\
\hline \multirow{2}{*}{$\mathrm{C}$} & & Verificação & $\begin{array}{l}\text { Verificar se o bloqueio foi } \\
\text { efetivo }\end{array}$ \\
\hline & & (Bloqueio efetivo) & \\
\hline \multirow{2}{*}{ A } & & Padronização & $\begin{array}{c}\text { Previnir contra o reaparecimento } \\
\text { do problema }\end{array}$ \\
\hline & & Conclusão & $\begin{array}{c}\text { Documentar todo o processo } \\
\text { para recuperação futura }\end{array}$ \\
\hline
\end{tabular}

Fonte: Adaptado de Campos (2004).

1. Identificação do problema: identificar o problema com maior relevância no setor estudado, o seu impacto econômico bem como, prejuízos do ponto de vista de insatisfação do cliente. 
2. Observação do problema: investigar as características específicas do problema por meio de uma ampla gama de pontos de vista diferentes. Para isso foram realizadas visitas diárias ao setor de wafer, informações foram coletadas a fim de compreender bem o problema encontrado. Aqui o brainstorming foi usado em duas subfases:

(a) Fase de Geração de ideias: os convidados foram motivados a pensar sobre as causas referentes ao número elevado de paradas de linha não programadas no setor de wafer por meio de uma revisão das etapas do processo de produção dos biscoitos. Assim que algum participante tinha alguma ideia, de imediato as informações (possíveis causas) foram digitadas e projetadas para a visualização de todos os presentes na reunião. A fase 1 foi dada como encerrada quando todos os participantes concordaram que não havia mais ideias para contribuir com o projeto.

(b) Fase de pontuação das ideias: os participantes atribuíram um peso referente ao grau de importância de cada possível causa levantada utilizando a escala likert: 1 (pouco influente), 3 (influência mediana) e 5 (muito influente).

3. Análise: as causas fundamentais (causas básicas, causa raiz) do problema foram discutidas.

4. Plano de ação: Um plano de ação foi elaborado a fim de minimizar ou eliminar as causas fundamentais identificadas no passo anterior.

5. Ação: a equipe atuou de maneira efetiva para eliminar as causas fundamentais do problema tratado. Todos os colaboradores foram previamente treinados a fim de garantir a eficiência na execução do plano de ação.

6. Verificação: foi verificado se as ações foram eficientes trazendo resultados positivos para a empresa.

7. Padronização: foram elaborados os procedimentos operacionais necessários para a padronização do tempo útil de manutenção na linha de produção estudada a fim de garantir a disponibilidade das máquinas, bem como melhorando sua eficiência.

8. Conclusão: Após concluir o projeto, foi realizada uma reunião de resultados, a fim de demonstrar a eficácia da metodologia utilizada bem como os resultados significativos alcançados. 


\section{Estudo de caso}

O estudo de caso foi realizado na unidade localizada no agreste de Pernambuco. Dentre seus produtos estão biscoitos laminados e recheados, massas, salgadinhos, massas para bolos e cafés. Neste trabalho, o estudo foi limitado à produção de biscoito wafer, produzindo diversos sabores. Atualmente, o setor de fabricação de wafer possui três linhas de produção, mas apenas duas linhas funcionam por vez.

A coleta de dados na linha de produção W03 foi feita em folha de verificação diariamente, antes e depois da implementação do MASP, por um período de três semanas entre agosto e setembro de 2019 e outras três semanas entre outubro e novembro de 2019. Os históricos das paradas de linha programadas e com os relatórios de paradas de linha não programadas foram analisadas.

\section{Resultados e discussões}

\subsection{Fase 1 - Identificação do problema}

Inicialmente, foi levantada a quantidade de vezes que a linha W03 do setor teve paradas não programadas e foram subdividas de acordo com a classificação das paradas, ou seja, parada para acompanhamento, corretiva, melhoria, operacional, preventiva ou setup. A Figura 3 apresenta os dados coletados de Agosto e Setembro de 2019.

Figura 3 - Histograma com os dados de paradas de linha W03

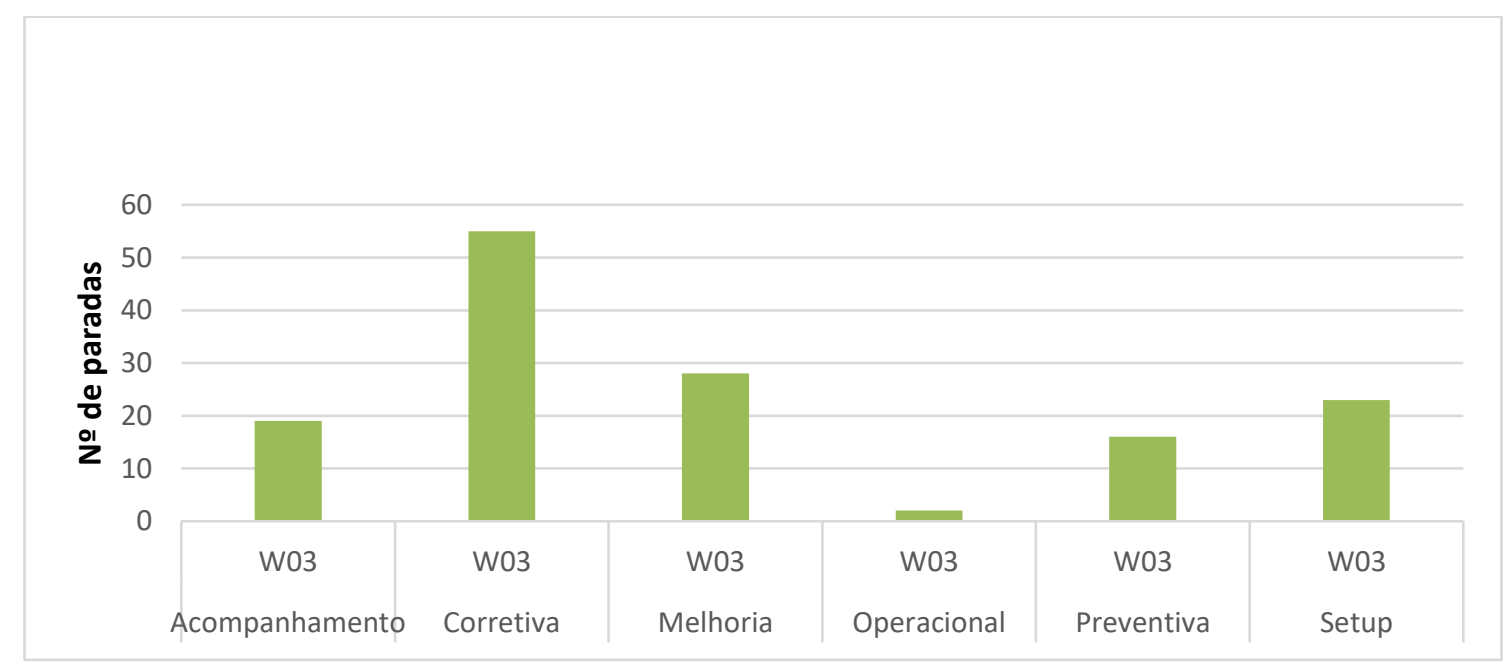

Fonte: Os autores (2019)

Conforme ilustrado, na Figura 3, a linha apresenta problemas relacionados à manutenção corretiva. A fim de identificar os setores em que ocorriam as maiores 
paradas na linha de produção um gráfico de Pareto foi feito com os dados coletados antes do MASP (Figura 4).

Figura 4 - Gráfico de Pareto dos setores que ocorreram as maiores paradas

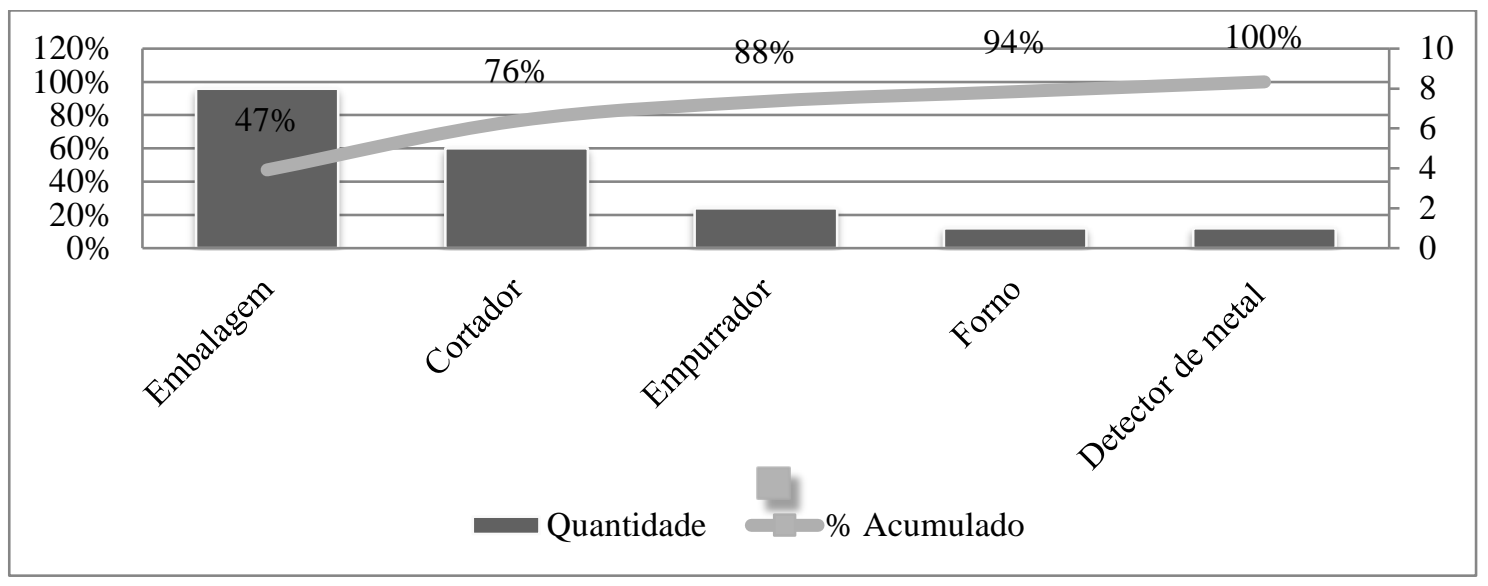

Fonte: Os autores (2019)

Observa-se (Figura 4) que a maior influência sobre as paradas não programadas foi na embalagem. Como consequência, a Tabela 1 apresenta o quantitativo de reprocessos nestes setores no mesmo período.

Tabela 1 - Dados do reprocesso da linha W03

\begin{tabular}{|l|r|r|r|}
\hline & Quantidade (KG) & & \% Acumulado \\
\hline Rocal & $3.376,20$ & $39 \%$ & $39 \%$ \\
\hline Empacotadeira & $2.242,50$ & $26 \%$ & $65 \%$ \\
\hline Corte & $1.546,60$ & $18 \%$ & $83 \%$ \\
\hline Recheadeira & 958,90 & $11 \%$ & $94 \%$ \\
\hline Casquinha & 516,70 & $6 \%$ & $100 \%$ \\
\hline Total & $8.640,90$ & & \\
\hline
\end{tabular}

Fonte: Os autores (2019).

Pode-se notar (Tabela 1) que o setor de embalagem, onde ocorreu o maior número de paradas não programadas, também foi responsável pelo maior número de reprocessos.

\subsection{Fase 2 - Observação do problema}

Nesta fase foi realizado um Brainstorming com colaboradores da empresa, desde operadores, supervisores até coordenadores. Dessa forma, as ideias/informações foram levantadas a fim de obter o máximo de possibilidades para solucionar o problema.

Antes de iniciar a reunião para a geração de ideias foi explanado e revisado rapidamente sobre o problema a qual se estava trabalhando. Foi ressaltado, também, a importância de 
todos, bem como suas ideais para análise. A Tabela 2 apresenta a pontuação relatada pelos participantes para cada causa identificada.

Tabela 2 - Quadro de pontuação do Brainstormin

\begin{tabular}{|c|c|c|c|c|c|c|c|c|}
\hline Número de causas que podem estar provocando o problema & \multicolumn{7}{|c|}{ Notas dos Participantes } & Média \\
\hline FALTADE TREINAMENTOS DOS COLABORADORES & 1 & 3 & 3 & 3 & 1 & 3 & 3 & 24 \\
\hline FALTA DE COMPROMENTIMENTO DOS COLABORADORES & 3 & 3 & 3 & 3 & 1 & 3 & 1 & 2,4 \\
\hline VARIAÇÃO DA MASSA & 3 & 3 & 3 & 3 & 5 & 5 & 5 & 3,8 \\
\hline FALHA NA COMUNICAÇÃO & 3 & 3 & 3 & 3 & 3 & 5 & 3 & 3,2 \\
\hline ELEVADO TEMPO DE TRANPORTE OU MOVIMENTAÇÃO DE MATERIA PRIMA & 3 & 3 & 3 & 3 & 3 & 3 & 3 & 3 \\
\hline EXCESSO DE CALOR NO SETOR DE EMBALAGEM & 4 & 5 & 4 & 4 & 4 & 3 & 5 & 4 \\
\hline FALTA DE MANUTENÇÃO PREVENTIVA & 5 & 5 & 5 & 5 & 5 & 5 & 5 & 5 \\
\hline QUEBRAS E MANUTENÇÃO CORRETIVAS & 5 & 5 & 5 & 5 & 5 & 5 & 5 & 5 \\
\hline ELEVADO TEMPO DE SETUP & 5 & 5 & 5 & 5 & 3 & 5 & 5 & 4,7 \\
\hline RESTRICCÕES EM MAQUINAS E EQUIPAMENTOS & 3 & 3 & 3 & 3 & 1 & 1 & 3 & 2,4 \\
\hline FALTA DE PADRONIZAÇÃO NO PROCESSO & 3 & 3 & 1 & 3 & 5 & 5 & 3 & 3,3 \\
\hline FALHA NO CONTROLE DE QUALIDADE & 3 & 3 & 3 & 5 & 5 & 3 & 3 & 3,6 \\
\hline VARIAÇÃO NA ESPESURA & 1 & 1 & 1 & 1 & 3 & 1 & 1 & 1,3 \\
\hline
\end{tabular}

Fonte: Os autores (2019).

As possíveis causas levantadas no Branstorming foram estruturadas no diagrama de causa e efeito (ou diagrama de Ishikawa), como mostra a Figura 5.

Figura 5 - Diagrama de Causa e efeito

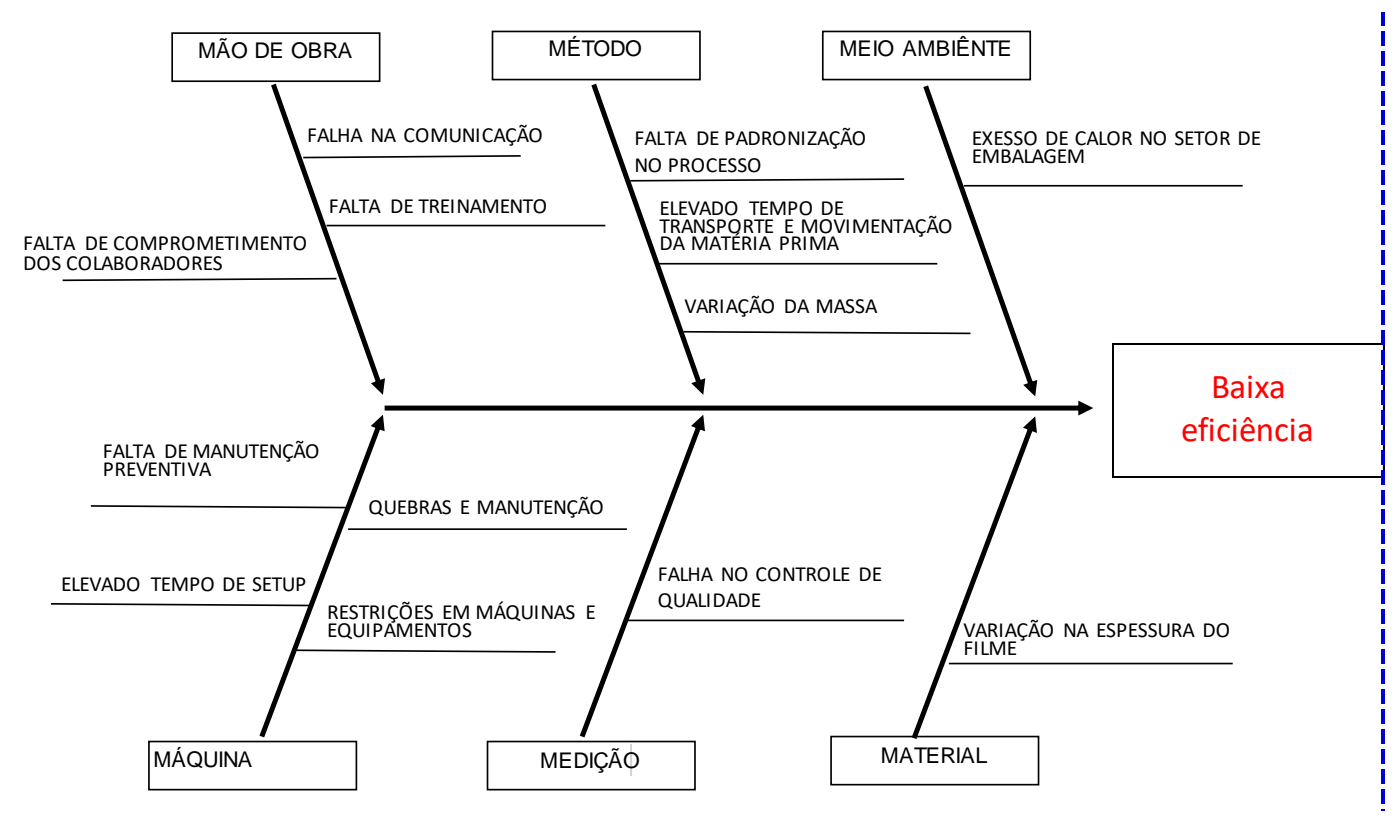

Fonte: Os autores (2019).

\subsection{Fase 3 - Análise do problema}

Após a elaboração do diagrama de Ishikawa foi realizada uma busca das sub-causas por meio da ferramenta dos 5 por quês, a fim de aumentar o nível de informação sobre o 
problema abordado, bem como um melhor entendimento, conforme ilustrado na Tabela 3.

Tabela 3 - Estudo dos por quês

\begin{tabular}{|c|c|c|c|c|c|c|}
\hline CAUSA & POR QUE? & POR QUE? & POR QUE? & POR QUE? & POR QUE? & CAUSA RAIZ \\
\hline $\begin{array}{c}\text { Quebras e } \\
\text { manutenção } \\
\text { corretiva } \\
\end{array}$ & $\begin{array}{c}\text { Ajuste da } \\
\text { maquina errado }\end{array}$ & $\begin{array}{l}\text { Operadores } \\
\text { diferentes }\end{array}$ & $\begin{array}{c}\text { Falta de } \\
\text { treinamenteo e } \\
\text { POP's }\end{array}$ & & & $\begin{array}{l}\text { Falta de } \\
\text { treinamento }\end{array}$ \\
\hline $\begin{array}{c}\text { Troca de } \\
\text { produto } \\
\text { durante o ciclo }\end{array}$ & $\begin{array}{c}\text { Programação da } \\
\text { produção }\end{array}$ & $\begin{array}{c}\text { Por não haver a } \\
\text { necessidade de } \\
\text { rodar o ciclo } \\
\text { completo com } \\
\text { um só produto }\end{array}$ & Baixo PVP & $\begin{array}{l}\text { Demanda } \\
\text { comercial }\end{array}$ & & $\begin{array}{l}\text { Planejamneto } \\
\text { da produção }\end{array}$ \\
\hline $\begin{array}{c}\text { Falta de } \\
\text { padronização } \\
\text { na preparação } \\
\text { da receita }\end{array}$ & $\begin{array}{c}\text { Os operadores } \\
\text { não seguem um } \\
\text { padrão na } \\
\text { mistura dos } \\
\text { ingredientes }\end{array}$ & $\begin{array}{c}\text { Não existe um } \\
\text { proceimento } \\
\text { para a mistura } \\
\text { dos } \\
\text { ingredientes } \\
\text { "MODO DE } \\
\text { PREPARO" }\end{array}$ & & & & $\begin{array}{l}\text { Não existe um } \\
\text { procedimento } \\
\text { padrão para a } \\
\text { mistura dos } \\
\text { ingredientes }\end{array}$ \\
\hline $\begin{array}{c}\text { Falta de } \\
\text { manutenção } \\
\text { preventiva }\end{array}$ & $\begin{array}{c}\text { Falta de } \\
\text { comunicação } \\
\text { entre os setores }\end{array}$ & $\begin{array}{c}\text { Falta de } \\
\text { proficionais } \\
\text { para fazer esses } \\
\text { planejamento }\end{array}$ & & & & $\begin{array}{c}\text { Falta de } \\
\text { planejamento }\end{array}$ \\
\hline
\end{tabular}

Fonte: Os autores (2019)

\subsection{Fase 4 - Plano de ação}

Após as análises fundamentais das causas sobre o problema trabalhado, elaborou-se um plano de ação. Esse plano de ação foi realizado tomando como base as informações obtidas pelos operadores, bem como dos líderes do setor em conjunto com os supervisores e coordenadores da área, e está exemplificada na Tabela 4.

Tabela 4 - Plano de ação

\begin{tabular}{c|l|c|c|c}
\hline Item & \multicolumn{1}{|c|}{ Atividades } & Resp. & Envolvidos & Status \\
\hline 1 & Falta de treinamento especializado na equipe & Func.1 & Produção e Manutenção & Ok \\
& Realizar cursos e palestras de capacitação & & & Ok \\
1.3 & para os líderes & Func.1 & Produção & Ok \\
1.4 & Elaborar 5S no setor & Func.1 & Produção e Manutenção & Realizar \\
1.5 & Elaborar POP's para o setor & Func.1 & Produção e Operação & Ok \\
& Realizar manutenção preventiva e registro & & & Ok \\
2 & das atividades & Func.1 & Produção e Manutenção & Ok \\
2.1 & Climatizar o setor de trabalho & Func.1 & Produção e Manutenção & Realizar \\
3 & Realizar um checklist do setor & Func.1 & Produção e Operação & Realizar \\
\hline
\end{tabular}




\subsection{Fase 5 - Execução}

As execuções das ações necessárias foram acompanhadas constantemente por meio de reuniões periódicas. Com todo o empenho da equipe, as ações foram muito bem realizadas com um custo relativamente baixo. No entanto, algumas delas ainda não foram realizadas dentro do prazo desta pesquisa, mas já tem uma data para a realização das mesmas.

\subsection{Fase 6 - Verificação}

Foi feita uma análise durante três semanas após a manutenção preventiva e notou-se uma redução significativa das paradas de linha e do reprocessamento dos biscoitos. A Figura 6 e a Tabela 5 mostram que a quantidade de paradas não programadas foi reduzida em 53\%, em comparação com os dados apresentados na Figura 4 e a Tabela 1 , assim como a quantidade de reprocesso gerado que reduziu em $65 \%$, respectivamente.

Figura 6 - Quantidade de paradas após as ações

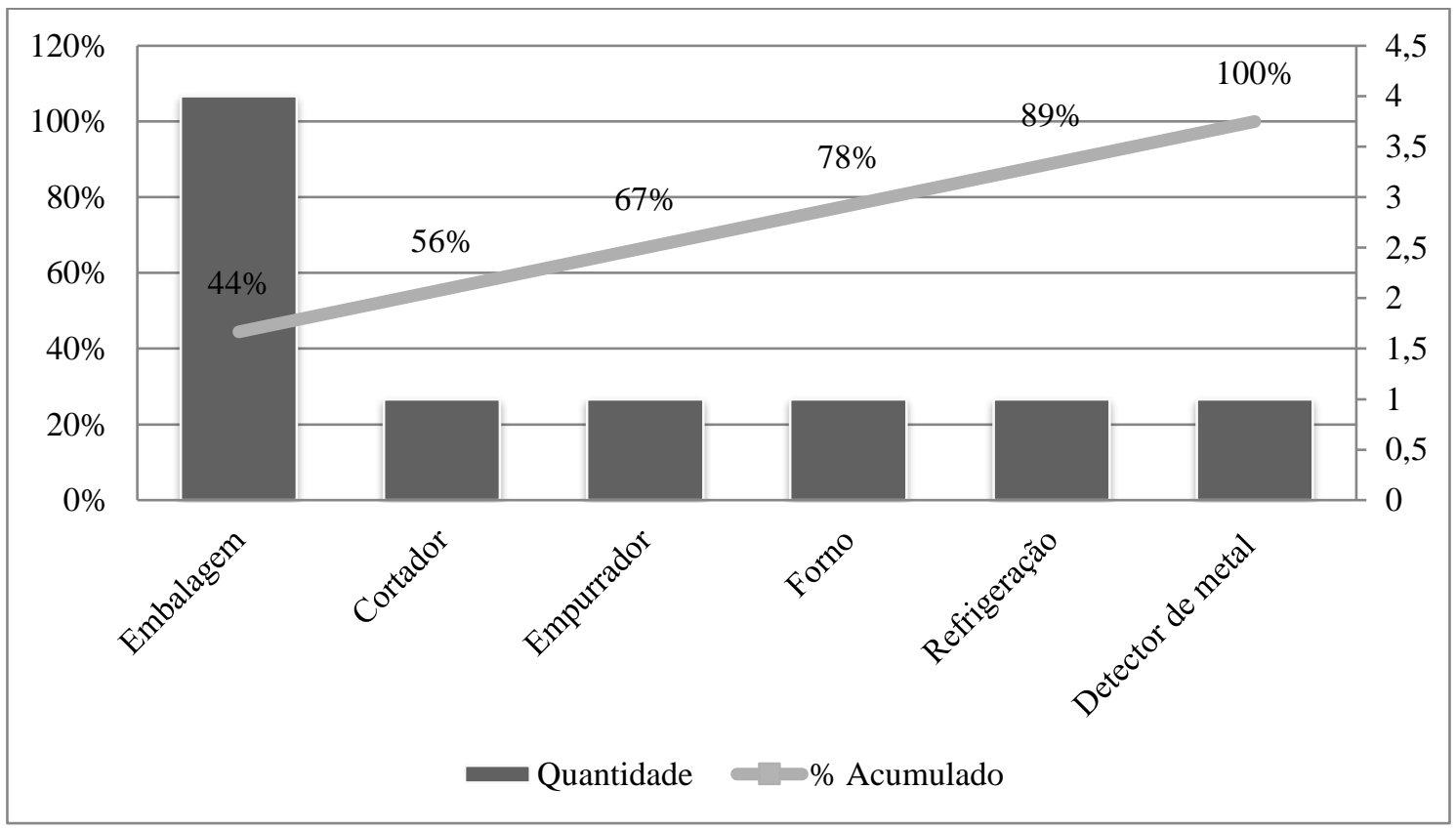

Fonte: Os autores (2019). 
Tabela 5 - Quantidade de reprocesso após as ações

\begin{tabular}{|l|r|r|r|}
\hline Local & Quantidade (KG) & $\%$ & $\%$ Acumulado \\
\hline Refugo & $1.104,50$ & $37 \%$ & $37 \%$ \\
\hline Empacotadeira & 895,40 & $30 \%$ & $67 \%$ \\
\hline Corte & 529,40 & $18 \%$ & $85 \%$ \\
\hline Recheadeira & 352,70 & $12 \%$ & $96 \%$ \\
\hline Casquinha & 105,40 & $4 \%$ & $100 \%$ \\
\hline Total & $2.987,40$ & & \\
\hline
\end{tabular}

Fonte: Os autores (2019).

\subsection{Fase 7 - Padronização}

Para que as paradas da produção não voltassem a ocorrer, foi fundamental fazer um treinamento e acompanhamento com os colaboradores, assim as ações que foram implantadas neste projeto devem fazer parte da rotina do dia a dia dos funcionários da operação.

\subsection{Fase 8 - Conclusão}

Analisando os resultados, pode-se observar o antes e o depois das paradas em todos os setores. Observa-se um resultado alcançado de 53\% de redução nas paradas de linha não programadas na linha W03 do setor de wafer.

\section{Conclusão}

Neste trabalho foi utilizado o método de análise e solução de problemas (MASP) por meio da aplicação de várias ferramentas da qualidade, para solucionar os problemas de paradas de linhas não programadas na empresa estudada, mais especificamente as perdas de biscoitos no setor de Wafer. As perdas originadas foram derivadas a um conjunto de fatores que se interligam, contribuindo de maneira significativa para um alto volume de paradas de linha, bem como em um alto tempo de retomada a produção.

Os resultados obtidos com esse estudo mostraram um ganho em temos de produtividade para a empresa bem como, uma visão mais holística em gestão da produção. A avaliação dos gestores quanto ao trabalho foi positiva em relação ao projeto proposto, 
sobretudo, o aprimoramento e a aplicabilidade de outras ferramentas para o setor, com o objetivo de manter um padrão modelo para a empresa.

Em suma, o presente trabalho proporcionou uma compreensão prática sobre os desafios enfrentados do dia a dia em uma indústria pelo profissional de engenharia de produção ao produzir um grande volume de produtos gerando o mínimo de perdas possíveis. Este trabalho proporcionou ainda, um conhecimento prático sobre a aplicabilidade de várias ferramentas da qualidade e a relação sobre cada uma delas para o problema tratado.

\section{REFERÊNCIAS}

ABIA. Associação Brasileira das Indústrias da Alimentação. A Força do Setor de Alimentos, 2017. Disponível em: https://www.abia.org.br/vsn/. Acesso em 25 de setembro de 2019.

CAMARGO, Wellington. Controle de Qualidade Total. IFPR, Curitiba, 2011.

CAMPOS, Vicente Falconi. TQC - Controle da Qualidade Total (no estilo japonês). Belo Horizonte: Ed. INDG Tecnologia e Serviços, 2004.

CARPINETTI, Luiz Cesar Ribeiro. Gestão da qualidade: conceitos e técnicas. - 2. ed. - São Paulo: Atlas, 2012.

DA SILVA, Valquiria; AMARAL, Ana Maria Pereira. Segurança Alimentar, Comércio Internacional e Segurança Sanitária. Informações Econômicas. 2004, vol.34, n.6, pp. 38-45.

DE AZEVEDO, Thayane Nascimento; COSTA, Rodrigo de Souza; SILVA, Ruy Gomes. A aplicação da etapa p do ciclo PDCA em uma empresa metalúrgica para redução de perdas e aumento da produtividade. XXXVIII Encontro Nacional de Engenharia de Produção. Maceió, Alagoas, 2018.

CHEN, Chialin; ZHANG, Jun; DELAURENTIS, Teresa. Quality control in food supply chain management: An analytical model and case study of the adulterated milk incident in China. International Journal of Production Economics. 2014, vol. 152, pp. 188-199.

LIMA, Luana Pereira; DO NASCIMENTO, Rane Gomes; FARIAS, Wagner da Silva. Influência da globalização nos hábitos culturais: aprendizagem significativa a partir da relação teoria-prática. Simpósio Internacional de Educação e Comunicação - SIMEDUC. 2016, vol. 9, n.1.

MARINO, Lúcia Helena Fazzane de Castro. Gestão da qualidade e gestão do conhecimento: fatoreschave para produtividade e competitividade empresarial. XIII Simpósio de Engenharia de Produção SIMPEP - Bauru, SP, 2006.

MARSHALL JR., Isnard ; ROCHA, Alexandre Varanda; MOTA, Edmarson Bacelar; QUINTELLA, Odair Mesquita Gestão da Qualidade e processos. Editora: FGV, 2012. 204p.

MACHADO, Simone Silva. Gestão da Qualidade. - Inhumas: IFG; Santa Maria: Universidade Federal de Santa $\quad$ Maria, 2012. $92 \quad$ p. Disponível em: <http://redeetec.mec.gov.br/images/stories/pdf/eixo_prd_industr/tec_acucar_alcool/161012_gest_qual.pdf $>$. Acesso em: 05 de nov de 2019.

MARA, Angélica de Souza; MARQUES, Darlan; ALVES, Jordania Louse Silva. Aplicação da ferramenta OEE: estudo de caso em uma indústria láctea do sudoeste goiano, XXXVIII Encontro Nacional de Engenharia de Produção - ENEGEP. 2018

PIRES, José Geraldo Carlos. Aprendizagem Organizacional através da Metodologia de Solução de Problemas - MASP. Revista de Administração da Fatea. 2014, vol. 9, n. 9, pp. 84-100. 
SEBRAE. Serviço Brasileiro de Apoio às Micro e Pequenas Empresas. Saiba o que é e como funciona a metodologia PDCA, [2020]. Disponível em https://www.sebrae.com.br/sites/PortalSebrae/artigos/4etapas-do-pdca-melhoram-gestao-dos-processos-e-qualidade-do-

produto,9083438af1c92410VgnVCM100000b272010aRCRD. Acesso em 07 de fevereiro de 2020.

SLACK, Nigel; CHAMBERS, Stuart; JOHNSTON, Robert. Administração da Produção. 4. ed. São Paulo: Atlas S.A., 2015.

VIEIRA, Sonia. Estatística para a qualidade. 3. Ed. Rio de Janeiro: Elsevier, 2014. 